and Systems

Manuscript Number:

Title: Positive Definite Indistinguishability Operators

Article Type: Full Length Article (FLA)

Keywords: t-norm; Continuous Archimedean t-norm; Additive generator; \$T\$indistinguishability operator; distance; positive definite matrix; Cayley-Menger determinant

Corresponding Author: Dr. Jordi Recasens, Ph.D

Corresponding Author's Institution: Universitat Politecnica de Catalunya

First Author: Jordi Recasens, Ph.D

Order of Authors: Jordi Recasens, Ph.D; Maria Santos Tomas 
Two geometric characterizations of positive definite reflexive and symmetric fuzzy relations are provided based on the embeddability of their naturally associated pseudodistances with respect to the continuous Archimedean $t$-norms with additive generators $t(x)=\arccos x$ and $t(x)=\operatorname{sqrt}(1-x)$.

Due to the important role of the $t$-norm $T_{-}$arccos $x$ with additive generator $t(x)=\arccos x$ in these characterizations, different characterizations of it are given. 


\title{
Positive Definite Indistinguishability Operators
}

\author{
J. Recasens \& M.S. Tomás \\ Department of Architectural Technology \\ ETSAV, ETSAB \\ UPC \\ Spain \\ j.recasens@upc.edu, maria.santos.tomas@upc.edu
}

\begin{abstract}
Two geometric characterizations of positive definite reflexive and symmetric fuzzy relations are provided based on the embeddability of their naturally associated pseudodistances with respect to the continuous Archimedean t-norms with additive generators $t(x)=\arccos x$ and $t(x)=\sqrt{1-x}$.

Due to the important role of the t-norm $T_{\arccos x}$ with additive generator $t(x)=\arccos x$ in these characterizations, different characterizations of it are given.

Keywords: t-norm, continuous Archimedean t-norm, additive generator, $T$-indistinguishability operator, distance, positive definite matrix, Cayley-Menger determinant.
\end{abstract}

\section{Introduction}

In Volume 157 of this journal two papers $([8,13])$ were devoted to the relationship between positive definiteness and transitivity of a reflexive and symmetric fuzzy relation from two different points of view. In [13] the subject was the study of usual similarity measures used e.g. in combinatorial chemistry while in [8] the interest was put on characterizing kernels (see also [9]). One of the main results shared by them is that a reflexive and symmetric fuzzy relation is three-positive semidefinite (see Definition 3.2) if it is 
$T_{\text {arccos }}$-transitive (i.e.: a $T_{\text {arccos }}$-indistinguishability operator) where $T_{\text {arccos }}$ is the continuous Archimedean t-norm with additive generator $t(x)=\arccos x$. Also the relationship between positive definiteness and the t-norm generated by $t(x)=\sqrt{1-x}$ was analyzed in [13]. Transitivity with respect to this tnorm was also considered in the study of fuzzy partitions in another paper of the same volume of this journal [4]. Lately, also in this journal, [3] insisted in the question and considered the open problem of characterizing the reflexive and symmetric fuzzy relations that are positive definite.

It is indeed an interesting problem and the present paper provides geometric characterizations of such fuzzy relations. After a section of preliminaries, Section 3 studies and characterizes in different ways the important continuous Archimedean t-norm $T_{\arccos }$ with additive generator $t(x)=\arccos x$ basically relating it to the vanishing of the determinant of one-dimensional $T$-indistinguishability operators (see Definition 3.5). The results of Section 4 provide characterizations of a positive definite reflexive and symmetric fuzzy relation $A$ based on the embeddability of the pseudodistances associated to $A$ (Proposition 2.9) by $T_{\sqrt{1-x}}$ and $T_{\text {arccos }}$ into a Euclidean space and a hypersphere respectively.

\section{Preliminaries}

This section contains the basic definitions and properties of t-norms, $T$-indistinguishability operators and positive definite matrices that will be used throughout the paper starting with the characterization of continuous Archimedean t-norms by their additive generators.

Proposition 2.1. [7] A t-norm $T$ is continuous Archimedean if and only if there exists a continuous decreasing function $t:[0,1] \rightarrow[0, \infty]$ with $t(1)=0$ such that for all $x, y \in[0,1]$

$$
T(x, y)=t^{[-1]}(t(x)+t(y))
$$

where $t^{[-1]}$ is the pseudo-inverse of $t$ defined by

$$
t^{[-1]}(x)= \begin{cases}t^{-1}(x) & \text { if } x \in[0, t(0)] \\ 0 & \text { otherwise. }\end{cases}
$$

$T$ is strict if $t(0)=\infty$ and non-strict otherwise. $t$ is called an additive generator of $T$ and two additive generators of the same t-norm differ only by a positive multiplicative constant. 
From a left-continuous t-norm its residuation and birresiduation can be derived. If the t-norm is used for modelling the logic conjunction, its residuation and birresiduation represent the logical implication and biimplication.

Definition 2.2. [7] Let $T$ be a left-continuous t-norm.

- The residuation $\vec{T}$ of $T$ is the map $\vec{T}:[0,1] \times[0,1] \rightarrow[0,1]$ defined for all $x, y \in[0,1]$ by

$$
\vec{T}(x, y)=\sup \{\alpha \in[0,1] \mid T(x, \alpha) \leq y\} .
$$

- The birresiduation $\overleftrightarrow{T}$ of $T$ is the map $\overleftrightarrow{T}:[0,1] \times[0,1] \rightarrow[0,1]$ defined for all $x, y \in[0,1]$ by

$$
\overleftrightarrow{T}(x, y)=\min (\vec{T}(x, y), \vec{T}(y, x))
$$

Proposition 2.3. [7] Let $T$ be a continuous Archimedean t-norm with additive generator $t$. Then for all $x, y \in[0,1]$

$$
\begin{aligned}
& \vec{T}(x, y)=t^{[-1]}(t(y)-t(x)) \\
& \overleftrightarrow{T}(x, y)=t^{-1}(|t(x)-t(y)|)
\end{aligned}
$$

We will use the following result that states that a left-continuous t-norm can be recovered from its residuation.

Proposition 2.4. [1] A t-norm is left-continuous if and only if $\inf \{\alpha \in$ $[0,1] \mid \vec{T}(x, \alpha) \geq y\}=T(x, y)$ for all $x, y \in[0,1]$.

From this proposition a left-continuous t-norm can be recovered from its residuation and hence a t-norm is characterized by it. It is not the case for non left-continuous t-norms as shown in the following example.

Example 2.5. [1] Consider the two (non left-continuous) t-norms $T_{1}$ and $T_{2}$ defined by

$$
\begin{gathered}
T_{1}(x, y)= \begin{cases}0 & \text { if }(x, y) \in\left[0, \frac{1}{2}\right] \times\left[0, \frac{1}{2}\right] \\
\min (x, y) & \text { otherwise }\end{cases} \\
T_{2}(x, y)= \begin{cases}T_{1}(x, y) & \text { if }(x, y) \neq\left(\frac{1}{2}, \frac{1}{2}\right) \\
\frac{1}{2} & \text { if }(x, y) \neq\left(\frac{1}{2}, \frac{1}{2}\right) .\end{cases} \\
T_{1} \neq T_{2} \text { but } \vec{T}_{1}=\vec{T}_{2} .
\end{gathered}
$$


Indistinguishability operators are one of the most important types of fuzzy relations because they fuzzify the concepts of equivalence and equality. They have been studied extensively both theoretically and from the applications point of view and the reader is referred to [10] for a general panorama.

Definition 2.6. $[15,10]$ Let $T$ be a t-norm and $X$ a set. A fuzzy relation $E$ on $X$ is a $T$-indistinguishability operator if and only if for all $x, y, z \in X$

- $E(x, x)=1$ (Reflexivity)

- $E(x, y)=E(y, x)$ (Symmetry)

- $T(E(x, y), E(y, z)) \leq E(x, z)$ (T-transitivity).

If $E(x, y)=1$ if and only if $x=y$, then $E$ separates points.

Definition 2.7. A reflexive and symmetric fuzzy relation $E$ on $X$ is called a proximity or tolerance relation.

Indistinguishability operators are related to distances from different points of view. One that will be needed in Section 4 is exposed in Proposition 2.9.

Definition 2.8. Let $X$ be a set. A mapping $d: X \times X \rightarrow[0, \infty]$ is a pseudodistance or pseudometric if and only if for all $x, y, z \in X$

- $d(x, x)=0$

- $d(x, y)=d(y, x)$

- $d(x, y)+d(y, z)) \geq d(x, z)$

If $d(x, y)=0$ if and only if $x=y$, then $d$ is a distance or metric on $X$.

Proposition 2.9. [14] Let $T$ be a continuous Archimedean $t$-norm, $t$ an additive generator of $T$ and $X$ a set. A fuzzy relation $E$ on $X$ is a $T$ indistinguishability operator if and only if $t(E)$ is a pseudodistance on $X$. E separates points if and only if $t(E)$ is a distance.

Let us finally recall the definition of positive definite matrix.

Definition 2.10. A symmetric $n \times n$ real matrix $A$ is positive definite if $\vec{u}^{t} A \vec{u}>0$ for every non-zero vector $\vec{u} \in \mathbb{R}^{n}$ and positive semi-definite if $\vec{u}^{t} A \vec{u} \geq 0$. 
There are many characterizations of positive definiteness. The next proposition recalls a couple of them and can be found in any book of linear algebra.

Proposition 2.11. Let $A$ be a symmetric $n \times n$ real matrix. The following statements are equivalent.

- $A$ is positive definite.

- All eigenvalues of $A$ are positive.

- All principal minors of $A$ are positive, where the $k$-th principal minor of a matrix $A$ is the determinant of its upper-left $k \times k$ sub-matrix.

\section{Characterizations of $T_{\arccos }$}

As it was mentioned in the introductory section, the continuous Archimedean t-norm $T_{\arccos }$ with additive generator $t(x)=\arccos x$ plays an important role in the characterization of positive semidefinite symmetric fuzzy relations. For example, a symmetric fuzzy relation is three-positive semidefinite (Definition $3.2)$ if and only if it is a $T_{\text {arcos-indistinguishability operator. This makes this }}$ t-norm special and therefore it seems worth studying it in more detail. Also in Subsection 4.1 $T_{\text {arcos }}$ will be used for a geometric characterization of positive definite symmetric fuzzy relations. This section will provide characterizations of this t-norm by using one-dimensional indistinguishability operators (see Definition 3.5).

The next proposition describes explicitly the t-norm $T_{\text {arccos }}$ and its residuation and birresiduation.

Proposition 3.1. For all $x, y \in[0,1]$,

$$
\begin{aligned}
T_{\arccos }(x, y) & =\max (\cos (\arccos x+\arccos y), 0) \\
& =\max \left(x y-\sqrt{1-x^{2}} \sqrt{1-y^{2}}, 0\right) \\
\vec{T}_{\arccos }(x, y) & =\cos (\max (0, \arccos y-\arccos x)) \\
& = \begin{cases}1 & \text { if } x \leq y \\
x y+\sqrt{1-x^{2}} \sqrt{1-y^{2}} & \text { if } x>y .\end{cases}
\end{aligned}
$$




$$
\begin{aligned}
\overleftrightarrow{T}_{\arccos }(x, y) & =\cos (|\arccos x-\arccos y|) \\
& =\cos (\arccos x-\arccos y) \\
& =x y+\sqrt{1-x^{2}} \sqrt{1-y^{2}}
\end{aligned}
$$

Definition 3.2. [13] A reflexive and symmetric fuzzy relation $A$ on a set $X=\left\{x_{1}, x_{2}, \ldots, x_{n}\right\}$ of cardinality $n>2$ is three-positive semidefinite if for all $0 \leq i, j, k \leq n, i \neq j \neq k \neq i$ the submatrix of $A$

$$
\left(\begin{array}{lll}
1 & a_{i j} & a_{i k} \\
a_{i j} & 1 & a_{j k} \\
a_{i k} & a_{j k} & 1
\end{array}\right)
$$

is positive semidefinite, where $a_{i j}$ stands for $A\left(x_{i}, x_{j}\right)$.

In $[8,13]$ the following characterization of the three positive semidefinite tolerance relations is given.

Proposition 3.3. $[8,13]$ A reflexive and symmetric fuzzy relation $A$ on a set $X$ is three-positive semidefinite if and only if it is $T_{\arccos }$-transitive (i.e.: it is a $T_{\text {arccos-indistinguishability operator). }}$.

The previous proposition has nice geometric interpretations.

- If $A=\left(a_{i j}\right)_{i, j=1,2,3}$ is a definite positive $3 \times 3$ matrix, then it is the matrix of an inner product $\langle\cdot, \cdot\rangle$ and we can find three linearly independent vectors $\overrightarrow{u_{1}}, \overrightarrow{u_{2}}, \overrightarrow{u_{3}}$ with $a_{i j}=\left\langle\overrightarrow{u_{i}}, \overrightarrow{u_{j}}>. a_{i i}=1\right.$ means that these vectors are unitary and the angle determined by $\overrightarrow{u_{i}}$ and $\overrightarrow{u_{j}}$ is then $\arccos a_{i j}$. Proposition 3.3 says that in order to be possible the existence of these vectors, these angles must verify the triangle inequality; in other words, the sum of two of them must be greater than or equal to the other one.

- Another geometric interpretation can be obtained taking into account that $\arccos a_{i j}$ is also the length of the arc joining the heads of the vectors $\overrightarrow{u_{i}}$ and $\overrightarrow{u_{j}}$ with their tails in the origin of coordinates. So the matrix $A$ is positive semidefinite if and only if the lengths of the arcs joining the heads of the vectors satisfy the triangle inequality. This will be generalized in Subsection 4.2. 
The next proposition provides the most natural way to generate a $T$ indistinguishability operator from a given fuzzy subset of a universe $X$. It generalizes (fuzzifies) the fact that in the crisp case a (crisp) subset $A \subseteq X$ partitions $X$ in two parts: $A$ and its complementary $X-A$.

Proposition 3.4. $[14,10]$ Let $\mu$ be a fuzzy set of a universe $X$ and $T$ a left continuous $t$-norm. The fuzzy relation $E_{\mu}$ on $X$ defined for all $x, y \in X$ by

$$
E_{\mu}(x, y)=\overleftrightarrow{T}(\mu(x), \mu(y))
$$

is a T-indistinguishability operator.

Definition 3.5. [5, 10] A T-indistinguishability operator on $X$ of the form $E_{\mu}$ for some fuzzy set $\mu$ of $X$ is called one-dimensional.

Lemma 3.6. Let $T$ be a continuous non-strict Archimedean t-norm, $t$ a generator of $T$ and $\mu$ a fuzzy subsets of a set $X$ of finite cardinality. Then there exists a normalized fuzzy set $\nu$ such that $E_{\mu}=E_{\nu}$.

Proof. Consider $k=\max \{-t(\mu(x)) \mid x \in X\}$ and $\nu$ defined for all $x \in X$ by

$$
\nu(x)=t^{-1}(t(\mu(x))+k) .
$$

- $\nu$ is normalized: Let $x_{0} \in X$ be such that $k=-t\left(\mu\left(x_{0}\right)\right)$. Then $\nu\left(x_{0}\right)=t^{-1}\left(t\left(\mu\left(x_{0}\right)\right)-t\left(\mu\left(x_{0}\right)\right)=t^{-1}(0)=1\right.$.

- $E_{\nu}=E_{\mu}$ :

$$
\begin{aligned}
E_{\nu}(x, y) & =t^{-1}\left(\left|t\left(t^{-1}(t(\mu(x))+k)\right)-t\left(t^{-1}(t(\mu(y))+k)\right)\right|\right) \\
& =t^{-1}(|t(\mu(x))-t(\mu(y))|)=E_{\mu}(x, y) .
\end{aligned}
$$

Lemma 3.7. Let $T$ be a left continuous t-norm, $X$ a set of finite cardinality and $\mu$ a constant fuzzy subset of $X$. Then $E_{\mu}(x, y)=1$ for all $x, y \in X$ and therefore the rank of $E_{\mu}$ is $1\left(\operatorname{rank}\left(E_{\mu}\right)=1\right)$.

Proof. Trivial.

The next proposition characterizes the t-norm $T_{\text {arccos }}$ as the one for whom $\operatorname{det}\left(E_{\mu}\right)=0$ for all fuzzy subsets of a set of cardinality 3 . 
Proposition 3.8. Let $X=\left\{x_{1}, x_{2}, x_{3}\right\}$ be a set of cardinality 3 and $T$ a left continuous t-norm. $\operatorname{det}\left(E_{\mu}\right)=0$ for all fuzzy subsets $\mu$ of $X$ if and only if $T=T_{\text {arccos }}$.

Proof. Thanks to the previous lemma we can consider the fuzzy subset $\mu$ of $X$ normalized and, without loss of generality, of the form $\mu=(1, x, y)$ with $x, y \in[0,1]$ and $1 \geq x \geq y$. Then,

$$
E_{\mu}=\left(\begin{array}{ccc}
1 & x & \multicolumn{1}{|c}{y} \\
x & 1 & \overleftrightarrow{T}(x, y) \\
y & \overleftrightarrow{T}(x, y) & 1
\end{array}\right)=\left(\begin{array}{ccc}
1 & x & y \\
x & 1 & \vec{T}(x, y) \\
y & \vec{T}(x, y) & 1
\end{array}\right)
$$

$\operatorname{det}\left(E_{\mu}\right)=1+2 x y \vec{T}(x, y)-x^{2}-y^{2}-(\vec{T}(x, y))^{2}=0$ if and only if $\vec{T}(x, y)=$ $x y+\sqrt{1-x^{2}} \sqrt{1-y^{2}}$.

Being this true for all $x, y \in[0,1]$ with $x \geq y$, and thanks to Proposition 2.4 we have $T=T_{\text {arcos }}$.

Another way to put this result is expressed in the next proposition.

Proposition 3.9. Let $X=\left\{x_{1}, x_{2}, x_{3}\right\}$ be a set of cardinality 3 and $T$ a left continuous t-norm. $\operatorname{rank}\left(E_{\mu}\right)=2$ for all non-constant fuzzy subsets $\mu$ of $X$ if and only if $T=T_{\text {arccos }}$.

The following result is a generalization in one direction of this result.

Proposition 3.10. Let $X=\left\{x_{1}, x_{2}, \ldots, x_{n}\right\}$ be a finite set of cardinality $n \geq 2, \mu$ a non-constant fuzzy subset of $X$ and $E_{\mu}$ the one-dimensional $T_{\text {arccos }}$-indistinguishability operator on $X$ generated by $\mu$. Then $\operatorname{rank}\left(E_{\mu}\right)=2$.

Proof. Without loss of generality we can assume that $\mu=\left(1, a_{2}, a_{3}, \ldots, a_{n}\right)$ with $1 \geq a_{2} \geq a_{3}, \geq \ldots \geq a_{n}$ and we can write $\mu=\left(1, \cos b_{2}, \cos b_{3}, \ldots, \cos b_{n}\right)$. As $\mu$ is non-constant, $a_{n} \neq 1$ and hence $\cos b_{n} \neq 1$ and $\sin b_{n} \neq 0$. Then

$$
\operatorname{rank}\left(E_{\mu}\right)=\operatorname{rank}\left(\begin{array}{ccccc}
1 & a_{2} & a_{3} & \ldots & a_{n} \\
a_{2} & 1 & \vec{T}\left(a_{2}, a_{3}\right) & \ldots & \vec{T}\left(a_{2}, a_{n}\right) \\
a_{3} & \vec{T}\left(a_{2}, a_{3}\right) & 1 & \ldots & \vec{T}\left(a_{3}, a_{n}\right) \\
\vdots & \vdots & \vdots & \ddots & \vdots \\
a_{n} & \vec{T}\left(a_{2}, a_{n}\right) & \vec{T}\left(a_{3}, a_{n}\right) & \ldots & 1
\end{array}\right)=
$$


Subtracting to the $i$-th column, $i>1$, the first one multiplied by $\cos b_{i}$ we get that the rank is

$$
\begin{array}{r}
\operatorname{rank}\left(\begin{array}{ccccc}
1 & 0 & 0 & \ldots & 0 \\
\cos b_{2} & 1-\cos ^{2} b_{2} & \sin b_{3} \sin b_{2} & \ldots & \sin b_{n} \sin b_{2} \\
\cos b_{3} & \sin b_{3} \sin b_{2} & 1-\cos ^{2} b_{3} & \ldots & \sin b_{n} \sin b_{3} \\
\vdots & \vdots & \vdots & \ddots & \vdots \\
\cos b_{n} & \sin b_{n} \sin b_{2} & \sin b_{n} \sin b_{3} & \ldots & 1-\cos ^{2} b_{n}
\end{array}\right)= \\
1+\operatorname{rank}\left(\begin{array}{cccc}
1-\cos ^{2} b_{2} & \sin b_{3} \sin b_{2} & \ldots & \sin b_{n} \sin b_{2} \\
\sin b_{3} \sin b_{2} & 1-\cos ^{2} b_{3} & \ldots & \sin b_{n} \sin b_{3} \\
\vdots & \vdots & \ddots & \vdots \\
\sin b_{n} \sin b_{2} & \sin b_{n} \sin b_{3} & \ldots & 1-\cos ^{2} b_{n}
\end{array}\right)= \\
\left.\begin{array}{cccc}
\sin b_{2} & \sin b_{3} \sin b_{2} & \ldots & \sin b_{n} \sin b_{2} \\
\sin b_{3} \sin b_{2} & \sin b_{3} & \ldots & \sin b_{n} \sin b_{3} \\
\vdots & \vdots & \ddots & \vdots \\
\sin b_{n} \sin b_{2} & \sin b_{n} \sin b_{3} & \ldots & \sin ^{2} b_{n}
\end{array}\right)=
\end{array}
$$

Subtracting to the $i$-th column, $i<n$, the last one multiplied by $\frac{\sin b_{i}}{\sin b_{n}}$ (recall that $\sin b_{n} \neq 0$ ) we get that the rank of $E_{\mu}$ is

$$
1+\operatorname{rank}\left(\begin{array}{cccc}
0 & 0 & \ldots & \sin b_{n} \sin b_{2} \\
0 & 0 & \ldots & \sin b_{n} \sin b_{3} \\
\vdots & \vdots & \ddots & \vdots \\
0 & 0 & \ldots & \sin ^{2} b_{n}
\end{array}\right)=2
$$

As a corollary we obtain the following characterization of the t-norm $T_{\text {arccos }}$. 
Proposition 3.11. Let $T$ be a left-continuous t-norm and $X$ a finite set of cardinality $n>2 . T=T_{\arccos }$ if and only if $\operatorname{rank}\left(E_{\mu}\right)=2$ for all non-constant fuzzy subsets of $X$.

Proof.

$\Rightarrow$ ) Proposition 3.10 .

$\Leftrightarrow)$ Consider a t-norm $T$ different from $T_{\text {arccos }}$ and $\mu$ a fuzzy subset of $X$ of the form $\left(1, a_{2}, a_{3}, \ldots, a_{n}\right)$ with $1>a_{2}>a_{3}$ and with $\vec{T}\left(a_{2}, a_{3}\right) \neq$ $\vec{T}_{\arccos }\left(a_{2}, a_{3}\right)$. (Such $a_{2}, a_{3}$ exist thanks to Proposition 2.4). Then $\operatorname{det}\left(\begin{array}{ccc}1 & a_{2} & a_{3} \\ a_{2} & 1 & \overleftrightarrow{T}\left(a_{2}, a_{3}\right) \\ a_{3} & \overleftrightarrow{T}\left(a_{2}, a_{3}\right) & 1\end{array}\right)=\operatorname{det}\left(\begin{array}{ccc}1 & a_{2} & a_{3} \\ a_{2} & 1 & \vec{T}\left(a_{2}, a_{3}\right) \\ a_{3} & \vec{T}\left(a_{2}, a_{3}\right) & 1\end{array}\right)$

This determinant is different from 0 thanks to Proposition 3.8 and therefore $\operatorname{rank}\left(E_{\mu}\right) \geq 3$.

\section{Two Geometric Characterizations of PSD Reflexive and Symmetric Fuzzy Relations}

In this section we provide two characterizations of positive definite reflexive and symmetric fuzzy relations: in Subsection 4.1 related to the embeddability into an Euclidean space while in Subsection 4.2 related to the embeddability into the hypersphere $\mathbb{S}^{n}=\left\{\vec{v}=\left(x_{0}, x_{1}, \ldots x_{n}\right) \in \mathbb{R}^{n+1} \mid \sum_{i=0}^{n} x_{i}^{2}=1\right\}$ in $\mathbb{R}^{n+1}$ with center in the origin of coordinates $\overrightarrow{0}$ and radius 1 .

\subsection{Embedding into $\mathbb{R}^{n}$}

Definition 4.1. Let $X=\left\{x_{0}, x_{1}, \ldots, x_{n}\right\}$ be a finite set of cardinality $n+1$ and $d$ a distance on $X$. Denoting $d\left(x_{i}, x_{j}\right)$ by $d_{i j}$ for all $0 \leq i, j \leq n$, the 
Cayley-Menger determinant $C M\left(x_{0}, x_{1}, \ldots, x_{n}\right)$ is

$$
\left|\begin{array}{cccccc}
0 & 1 & 1 & 1 & \ldots & 1 \\
1 & 0 & d_{01}^{2} & d_{02}^{2} & \ldots & d_{0 n}^{2} \\
1 & d_{01}^{2} & 0 & d_{12}^{2} & \ldots & d_{1 n}^{2} \\
1 & d_{02}^{2} & d_{12}^{2} & 0 & \ldots & d_{2 n}^{2} \\
\vdots & \vdots & \vdots & \vdots & \ddots & \vdots \\
1 & d_{0 n}^{2} & d_{1 n}^{2} & d_{2 n}^{2} & \ldots & 0
\end{array}\right|
$$

The importance of the Cayley-Menger determinant is that if the set $X$ is in $\mathbb{R}^{n}$ and $d$ is the Euclidean distance, then it is related with the volume of the $n+1$-simplex generated by the points of $X$ in the following way.

Proposition 4.2. Let $X=\left\{x_{0}, x_{1}, \ldots, x_{n}\right\}$ be a finite set of $n+1$ points in $\mathbb{R}^{n}$ and $d$ the Euclidean distance. Then the volume $v(X)$ of the $n+1$-simplex with vertices the elements of $X$ is

$$
v(X)=\sqrt{\frac{(-1)^{n+1}}{2^{n}(n !)^{2}} C M\left(x_{0}, x_{1}, \ldots, x_{n}\right)} .
$$

The previous result can be found in every book and article devoted to Euclidean embeddings of metric spaces. One of the earliest ones, cited in [6], is [12]. In particular, the Cayley-Menger determinant must have the same sign as $(-1)^{n+1}$. From this, we have the following result that characterizes the finite metric spaces isometrically embeddable into an Euclidean space.

Proposition 4.3. [6] Let $X=\left\{x_{0}, x_{1}, \ldots, x_{n}\right\}$ be a finite set of cardinality $n+1$ and $d$ a distance on $X$. Denoting $d\left(x_{i}, x_{j}\right)$ by $d_{i j}$ for all $0 \leq i, j \leq n$, $(X, d)$ is embeddable into $\mathbb{R}^{n}$ if and only if for every $k=1,2, \ldots, n$ the sign of $C M\left(x_{0}, x_{1}, \ldots, x_{k}\right)$ is equal to $(-1)^{k+1}$.

Proposition 4.4. [11] Let $X=\left\{x_{0}, x_{1}, \ldots, x_{n}\right\}$ be a finite set of cardinality $n+1$ and $d$ a distance on $X$. Denoting $d\left(x_{i}, x_{j}\right)$ by $d_{i j}$ for all $0 \leq i, j \leq n$, $(X, d)$ is embeddable into $\mathbb{R}^{n}$ if and only if the $n \times n$ matrix with entries $x_{i j}=\frac{d_{0 i}^{2}+d_{0 j}^{2}-d_{i j}^{2}}{2}(1 \leq i, j \leq n)$ is positive definite.

In the case of a reflexive and symmetric fuzzy relation, its associated $n \times n$ matrix $A=\left(x_{i j}\right)$ has ones in its diagonal, so that for all $i=1,2, \ldots, n$, we have

$$
1=x_{i i}=\frac{d_{0 i}^{2}+d_{0 i}^{2}-d_{i i}^{2}}{2}=\frac{2 d_{0 i}^{2}}{2}=d_{0 i}^{2}
$$


and hence

$$
d_{0 i}=1 .
$$

So in this case the Cayley-Menger determinant of $d$ is

$$
\left|\begin{array}{cccccc}
0 & 1 & 1 & 1 & \cdots & 1 \\
1 & 0 & 1 & 1 & \cdots & 1 \\
1 & 1 & 0 & d_{12}^{2} & \cdots & d_{1 n}^{2} \\
1 & 1 & d_{12}^{2} & 0 & \cdots & d_{2 n}^{2} \\
\vdots & \vdots & \vdots & \vdots & \ddots & \vdots \\
1 & 1 & d_{1 n}^{2} & d_{2 n}^{2} & \cdots & 0
\end{array}\right|
$$

Moreover, from

$$
x_{i j}=\frac{d_{0 i}^{2}+d_{0 j}^{2}-d_{i j}^{2}}{2}=\frac{2-d_{i j}^{2}}{2}
$$

we get

$$
d_{i j}=\sqrt{2} \sqrt{1-x_{i j}} \text { for all } 1 \leq i, j \leq n .
$$

In terms of the elements of $A$, then

$$
C M\left(x_{0}, x_{1}, \ldots, x_{n}\right)=\left|\begin{array}{cccccc}
0 & 1 & 1 & 1 & \ldots & 1 \\
1 & 0 & 1 & 1 & \ldots & 1 \\
1 & 1 & 0 & 2\left(1-x_{12}\right) & \ldots & 2\left(1-x_{1 n}\right) \\
1 & 1 & 2\left(1-x_{12}\right) & 0 & \ldots & 2\left(1-x_{2 n}\right) \\
\vdots & \vdots & \vdots & \vdots & \ddots & \vdots \\
1 & 1 & 2\left(1-x_{1 n}\right) & 2\left(1-x_{2 n}\right) & \ldots & 0
\end{array}\right|
$$

and we obtain the following geometric characterization of reflexive and symmetric fuzzy relations with positive definite associated matrix.

Proposition 4.5. A reflexive and symmetric fuzzy relation $A$ on a set $X=$ $\left\{x_{1}, x_{2}, \ldots, x_{n}\right\}$ of finite cardinality is positive definite if and only if it is a $T_{\sqrt{1-x}}$-indistinguishability operator and in $X^{\prime}=X \cup\left\{x_{0}\right\}$ the associated distance $d$ (i.e.: $d_{i j}=\sqrt{2} \sqrt{1-x_{i j}}$ if $i, j>0$ and $d_{0 i}=1$ for $i>0$ ) is embeddable into $\mathbb{R}^{n}$.

In the embedding, $x_{0}$ can be sent to the origin of coordinates $x_{0}^{\prime}$ and the images $x_{i}^{\prime}$ of $x_{i}, i>0$ correspond to the heads of the vectors $\overrightarrow{x_{0}^{\prime} x_{i}^{\prime}}$ so that the last proposition can be stated in the a clearer way 
Proposition 4.6. A reflexive and symmetric fuzzy relation $A=\left(x_{i j}\right)$ on a set $X=\left\{x_{1}, x_{2}, \ldots, x_{n}\right\}$ of finite cardinality $n$ is positive definite if and only if it is a $T_{\sqrt{1-x}}$-indistinguishability operator and $X$ with the distance $d\left(x_{i}, x_{j}\right)=\sqrt{2} \sqrt{1-x_{i j}}, 1 \leq i, j \leq n$, is embeddable into $\mathbb{R}^{n}$ in such a way that the images of the points of $X$ lie on the hypersphere $\mathbb{S}^{n-1}$.

In particular, for a set $X$ of cardinality 3 this means that the determinant

$$
\begin{aligned}
& \left|\begin{array}{ccccc}
0 & 1 & 1 & 1 & 1 \\
1 & 0 & 1 & 1 & 1 \\
1 & 1 & 0 & 2\left(1-x_{12}\right) & 2\left(1-x_{13}\right) \\
1 & 1 & 2\left(1-x_{12}\right) & 0 & 2\left(1-x_{23}\right) \\
1 & 1 & 2\left(1-x_{13}\right) & 2\left(1-x_{23}\right) & 0
\end{array}\right|= \\
& 8\left(x_{12} x_{13} x_{23}-x_{12}^{2}-x_{13}^{2}-x_{23}^{2}+1\right)
\end{aligned}
$$

must be greater than 0 . This is equivalent to the fact that $A$ is $T_{\text {arccos }^{-}}$ transitive and Proposition 3.3 can be re-interpreted in the following way.

Proposition 4.7. The following statements for a reflexive and symmetric fuzzy relation $R$ on a set $X$ are equivalent.

- $R$ is three-positive semidefinite.

- $R$ is a $T_{\text {arccos-indistinguishability operator. }}$

- $\sqrt{1-R}$ is a pseudodistance on $X$ and every subset of cardinality 3 of $X$ is embeddable in $\mathbb{R}^{3}$ in such a way that the points of $X$ lie on the sphere $\mathbb{S}^{2}$.

This last proposition also shows the relation between $T_{\arccos }$ and $T_{\sqrt{1-x}}$. In [13] it has been proved that if a reflexive and symmetric fuzzy relation is three positive semidefinite, then it is $T_{\sqrt{1-x}}$-transitive. It is an interesting result that does not directly follow from the $T_{\text {arccos }}$-transitivity because the two tnorms are not comparable: $T_{\arccos }(0.7,0.8)=0.13>0.10=T_{\sqrt{1-x}}(0.7,0.8)$ and $T_{\text {arccos }}(0.8,0.8)=0.28<0.45=T_{\sqrt{1-x}}(0.8,0.8)$. The previous result clarifies this situation. 


\subsection{Embedding into $\mathbb{S}^{n}$}

While in the previous subsection the characterization of a positive definite tolerance relation $A$ has been obtained studying the embeddability of the metric generated from $A$ using the additive generator $t(x)=\sqrt{1-x}$ of the t-norm $T_{\sqrt{1-x}}$ into an Euclidean space, in this subsection the characterization will be done by studying the embeddability of the metric generated by the additive generator $t(x)=\arccos x$ of the $\mathrm{t}$-norm $T_{\arccos }$ into a hypersphere. This will generalize the geometric interpretations of Proposition 3.3.

Let $\mathbb{S}^{n}=\left\{\vec{v}=\left(x_{0}, x_{1}, \ldots x_{n}\right) \in \mathbb{R}^{n+1} \mid \sum_{i=0}^{n} x_{i}^{2}=1\right\}$ be the hypersphere in $\mathbb{R}^{n+1}$ with center in the origin of coordinates $\overrightarrow{0}$ and radius 1 . The spherical metric (or great circle metric) $d$ is the metric on $\mathbb{S}^{n}$ defined for all $\vec{u}=$ $\left(x_{0}, x_{1}, \ldots x_{n}\right), \vec{v}=\left(y_{0}, y_{1}, \ldots, y_{n}\right) \in \mathbb{S}^{n}$ by

$$
d(\vec{u}, \vec{v})=\arccos \left(\left|\sum_{i=0}^{n} x_{i} \cdot y_{i}\right|\right)=\arccos <\vec{u}, \vec{v}>
$$

where $\langle\vec{u}, \vec{v}\rangle$ is the standard inner product in $\mathbb{R}^{n+1}$. It is the length of the greatest circle arc joining $\vec{u}$ and $\vec{v}$.

Proposition 4.8. Let $X=\left\{x_{0}, x_{1}, \ldots, x_{n}\right\}$ be a finite set of cardinality $n+1$ and $d$ a distance on $X$. Denoting $d\left(x_{i}, x_{j}\right)$ by $d_{i j}$ for all $0 \leq i, j \leq n,(X, d)$ is embeddable in $\mathbb{S}^{n}$ if and only if the $n \times n$ matrix with entries $x_{i j}=\cos d_{i j}$ is positive definite.

In this case, the matrix $A$ with entries $x_{i j}$ is the matrix of a reflexive and symmetric fuzzy relation.

The Cayley-Menger determinant of $d$ is

$$
\left|\begin{array}{cccccc}
0 & 1 & 1 & 1 & \ldots & 1 \\
1 & 0 & 1 & 1 & \ldots & 1 \\
1 & 1 & 0 & \arccos ^{2} x_{12} & \ldots & \arccos ^{2} x_{1 n} \\
1 & 1 & \arccos ^{2} x_{12} & 0 & \ldots & \arccos ^{2} x_{2 n} \\
\vdots & \vdots & \vdots & \vdots & \ddots & \vdots \\
1 & 1 & \arccos ^{2} x_{1 n} & \arccos ^{2} x_{2 n} & \ldots & 0
\end{array}\right|
$$

Moreover,

$$
x_{i j}=\cos d_{i j} .
$$


So the next proposition provides an alternative geometric characterization of reflexive and symmetric fuzzy relations with positive definite associated matrix.

Proposition 4.9. A reflexive and symmetric fuzzy relation $A$ on a set $X=$ $\left\{x_{1}, x_{2}, \ldots, x_{n}\right\}$ of finite cardinality is positive definite if and only if it is

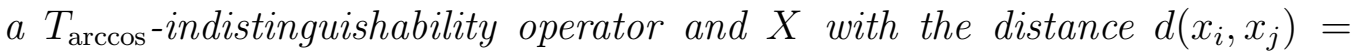
$\arccos x_{i j}, 1 \leq i, j \leq n$ can be mapped isometrically into $\mathbb{S}^{n-1}$ with the spherical metric.

\section{Concluding Remarks}

In this paper metric characterizations of positive definite reflexive and symmetric fuzzy relations have been provided using known results of embeddability into Euclidean spaces and hyperspheres with the associated distances generated by additive generators of $T_{\text {arccos }}$ and $T_{\sqrt{1-x}}$ according to Proposition 2.9 .

The results obtained in Subsection 4.2 permit a geometric elegant alternative proof of Propositions 3.8 and 3.10. A sketch of it follows: It is known that an indistinguishability operator $E$ separating points on a set $X$ with $E(x, y) \neq 0$ for all $x, y \in X$ with respect to a continuous Archimedean tnorm determines a metric betweenness relation [6] on $X$ that is linear if and only if $E$ is one dimensional [10]. In particular such a one dimensional $T_{\text {arccos}}{ }^{-}$ indistinguishability operator $E$ on $X=\left\{x_{1}, x_{2}, \ldots, x_{n}\right\}$ determines a linear metric betweenness relation on $X$. Then $t(E)=\arccos (E)$ is a distance that also determines a linear betweenness relation on $X[2]$ and hence the points of $X$ can be isometrically embedded into an arc of a hypersphere. Together with the center of this hypersphere they determine an $n+1$-simplex that lies on a plane and therefore any three of the vectors $\overrightarrow{x_{0} x_{1}}, \overrightarrow{x_{0} x_{2}}, \ldots, \overrightarrow{x_{0} x_{n}}$ are linearly dependent. (This can be also interpreted as that all the volumes of this simplex of dimension greater than 2 are 0 ).

The t-norm $T_{\sqrt{1-x}}$ belongs to the family of Yager t-norms [7] (it corresponds to the value $\frac{1}{2}$ of the parameter). Modifying the generator $t(x)=$ $\arccos x$ of the t-norm $T_{\text {arccos }}$, interesting families of t-norms can be obtained. Indeed, from a continuous, increasing function $f:[0,1] \rightarrow[0,1]$ with $f(1)=1$ we can consider the t-norm with additive generator $t(x)=\arccos (f(x))$. In 
particular, for $f_{\lambda}(x)=x^{\lambda}, 0<\lambda<\infty$ we have the family $\left(T_{\lambda}\right)_{\lambda \in(0, \infty)}$ of non-strict continuous Archimedean t-norms with additive generators $t_{\lambda}(x)=$ $\arccos x^{\lambda}$. It can be proved that $\lim _{\lambda \rightarrow \infty} T_{\lambda}$ is the drastic t-norm $T_{D}$ while $\lim _{\lambda \rightarrow 0} T_{\lambda}$ is the t-norm $T$ defined for all $x, y \in[0,1]$ by

$$
T(x, y)=\frac{x \cdot y}{\mathrm{e}^{2 \cdot \sqrt{\ln x \cdot \ln y}}}
$$

which is strict (its diagonal is $T(x, x)=x^{4}$ ). An additive generator of $T$ is $t(x)=\sqrt{-\ln x}$ as can be checked by a direct calculation or by considering that the limit of the sequence $s_{\lambda}(x)=\frac{1}{\sqrt{\lambda}} \cdot \arccos x^{\lambda}$ of additive generators of the family $\left(T_{\lambda}\right)_{\lambda \in(0, \infty)}$ when $\lambda$ tends to 0 is $\sqrt{2} \cdot \sqrt{-\ln x}$ and applying Corollary 8.21 of $[7]$.

\section{References}

[1] D. Boixader, Some Properties Concerning the Quasi-inverse of a t-norm Mathware \& Soft Computing 5 (1998) 5-12.

[2] D. Boixader, J. Recasens, Indistinguishability Operators with Respect to Different t-norms. Int. J. Uncertainty Fuzziness and Knowledge-based Systems 20 (2012) 167-183.

[3] C. Degang, Z. Deli, Structure of feature spaces related to fuzzy similarity relations as kernels. Fuzzy Sets and Systems 237 (2014) 90-95.

[4] L. Foulloy, E. Benoit, Building a class of fuzzy equivalence relations Fuzzy Sets and Systems 157(11) (2006) 1417-1437.

[5] J. Jacas, On the generators of T-indistinguishability operators. Stochastica 12 (1988) 49-63.

[6] K. Menger, Untersuchungen über allgemeine Metrik. Math. Ann. 100 (1928) 75-113.

[7] E.P., Klement, R. Mesiar, E. Pap, Triangular norms. Kluwer Academic Publishers, Dordrecht (2000).

[8] B. Moser, On the T-transitivity of kernels Fuzzy Sets and Systems 157(13) (2006) 1787-1796. 
[9] B. Moser, On representing and generating kernels by fuzzy equivalence relations, J. Mach. Learn. Res. 7 (2006) 2603-2620.

[10] J. Recasens, Indistinguishability Operators. Modelling Fuzzy Equalities and Fuzzy Equivalence Relations. Studies in Fuzziness and Soft Computing. Springer, 2011.

[11] I. J. Schoenberg, Remarks to Maurice Fréchet's article 'Sur la définition axiomatique d'une classe d'espace distanciés vectoriellement applicable sur l'espace de Hilbert', Ann. Math. 36 (1935) 724-732.

[12] P.H. Schouten, Mehrdimensionale Geometrie 2 (Die Polytope). Sammlung Schubert XXXVI, Leipzig (1905).

[13] M.S. Tomás, C. Alsina, J. Rubio-Martinez, Pseudometrics from threepositive semidefinite similarities Fuzzy Sets and Systems 157(17) (2006) $2347-2355$.

[14] L. Valverde, On the Structure of F-indistinguishability Operators. Fuzzy Sets and Systems 17 (1985) 313-328.

[15] L.A. Zadeh, Similarity relations and fuzzy orderings. Inform. Sci. 3 (1971) 177-200. 